\title{
Report on the 2018 Trials of the Multistatic NeXtRAD Dual Band Polarimetric Radar
}

\author{
M.R. Inggs and S. Lewis \\ Department of Electrical Engineering, \\ University of Cape Town, \\ Rondebosch, 7701, South Africa \\ Email: Michael.Inggs, Simon.Lewis at uct.ac.za
}

\author{
R.Palamà and M.A Ritchie and H. Griffiths \\ Department of Electronic \\ and Electrical Engineering \\ University College London \\ London EC1 7EJ UK \\ Email: r.palama, m.ritchie, h.griffiths at ucl.ac.uk
}

\begin{abstract}
NeXtRAD is a polarimetric, $L$ and $X$ Band, multistatic (three nodes), pulse Doppler radar, developed by UCT and UCL, as a follow on to the NetRAD sensor. This paper reports on the trials carried out in 2018, mostly in Simon's Bay, South Africa. The sensors (one active, two passive) are connected by WiFi communications link, with a maximum separation of $40 \mathrm{~km}$. Practically, results are reported with $8 \mathrm{~km}$ maximum baselines. The focus is on targets in sea clutter and microDoppler. We report on the final integration and test of the system command and control system that allows for scheduling of measurement and recording of bursts of pulses, as well as video of the radar field of view. Some innovations have been made in terms of digital hardware, firmware, and high performance computing technology. The system is synchronised with the UCT GPS Disciplined Oscillators (one per node), but we also report on bistatic measurements with White Rabbit, fibre timing system, as well as the consequences of GPS failure (GPS Denied Environment).
\end{abstract}

\section{INTRODUCTION}

\section{A. Background}

Multistatic radar has been a research interest of UCL and UCT for many years, firstly with the $\mathrm{S}$ Band NetRAD system [1], followed by the development of the dual band NeXtRAD system from the requirements spelled out in Inggs et al. [2]. Clearly the system aims at measuring multistatic target phenomenology, including land and sea clutter, but the systems have also provided opportunities to explore new technologies in the construction. For example, a thorough understanding of the timing between distant nodes is important [3]-[5], using GPS synchronisation with common view satellites, as well as optical fibre [6]. Data from NetRAD is available in a comprehensive database [7], soon to be followed by NeXtRAD. The flexible architecture opens up opportunities for investigating Symbiosis between Radar and Communications, networked sensors and the vulnerability of these to countermeasures and denial of synchronisation.

\section{B. Paper overview}

The paper begins with a brief overview of the NeXtRAD system as it now stands, including basic specifications and some of the complex command and control, firmware and software required to realise a distributed sensor with central control. Section III describes the setting to work of this complex, networked, sensor. The work planned and carried out during December 2018 is outlined in Sections IV,V. There will be many years of analysis required to harvest all the information in this data set, and some of the results achieved to date are given in section VI. The paper concludes with a discussion of future work.

\section{NEXTRAD SPECIFICATION AND CONFIGURATION}

\section{A. Architecture}

As shown in Figure 1, NeXtRAD is a three node, dual band, fully polarimetric sensor. Only one node is equipped with high power amplifiers, connected via a programmable frequency transceiver. The two passive nodes have only receiver downconverters from RF to a $125 \mathrm{MHz}$ IF. This is directly sampled by a Cobalt subsystem from Pentek. However, the digital waveform generator is present in all three nodes for possible future expansion of capability. In all three nodes.

In terms of polarimetry, there are two $\mathrm{X}$ Band analogue receive channels, so $\mathrm{HH}, \mathrm{HV}$ and $\mathrm{VV}, \mathrm{VH}$ are captured on alternating pulses. For L Band (which has lower Doppler requirements), a single receive channel means that a full polarimetric set takes four L Band transmissions to acquire. The cost of high power switches being prohibitive, the current system requires manual transmitter polarisation changes.

As shown in Figure 1 the nodes are connected by a WiFi network (5GHz Band), feeding to local switches. This means that the Command and Control Node $(\mathrm{C} \& \mathrm{C})$ is able to access the local laptop node controllers, as well as many of the subsystems that have network connections. This simplifies the transport of control instructions, but clearly leads to a potentially complex master software block, and devolved control. The system thus has simple, text files in a defined format (.ini), which are set up before the mission, or, altered on the fly. This single file is then sent to all nodes of the radar that then configure themselves ready for the data take. The time of acquisition is also set. After every data take, this header file is included into the database for that block of data.

We developed a quicklook software [8], [9] that allows operators to see the Range Time Intensity (RTI), rangeDoppler and spectrogram plots from the latest acquired data. This code, using threading, runs on the node controller. 


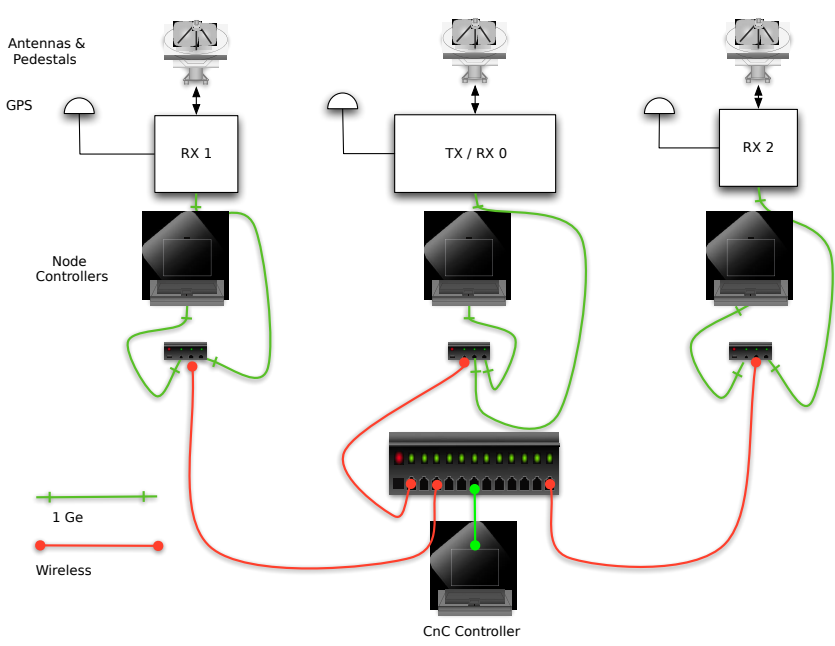

Fig. 1: Simplified system block diagram of NeXtRAD, showing one active, monostatic node, and two passive receiver-only nodes. Not shown are network TV cameras at each node that allow the node operator and system controller to view the antenna regard. In addition, a VOIP system running over the networks allows all operators to talk via headsets.

High performance computers utilise the Ansible [10] framework for control and distribution of tasking to nodes. This framework solved the problem for the system controller to distribute the Header Files to all nodes, and then to establish that all nodes are ready for acquisition. For each measurement the raw binary data is stored in a subdirectory that is named by date and time. Included is the executed header file, as well as the video recorded for the node.

At the end of each day, the data is scanned for key parameters per data take, and transferred to a database (MySQL). This database can be queried by researchers to find data sets conforming to requirements such as weather conditions, target type, bistatic angles, etc. After this, the contents of the directory are folded into an HDF5 structure for each data take. This software is still in development, so is being supplimented by a simple spreadsheet, summarising the measurements.

\section{B. Specification}

TABLE I: NeXtRAD specifications

\begin{tabular}{|l|l|l|}
\hline Parameter & Value & Comments \\
\hline X Band freq range & 8.5 to $9.2 \mathrm{GHz}$ & $\begin{array}{l}\text { In practice, limited } \\
\text { by power amplifier } \\
\text { to } 8500 \mathrm{MHz}\end{array}$ \\
L Band & 1.2 to $1.4 \mathrm{GHz}$ & $\begin{array}{l}\text { Full band possible. } \\
\text { 2 Receive channels }\end{array}$ \\
Polarimetry X Band & co- and cross-polar & 1 Receive channel \\
Polarimetry L Band & co- or cross-polar alt PRI & Both bands \\
Instantaneous BW & $50 \mathrm{MHz}$ & $10 \%$ Duty cycle \\
Peak power X Band & $400 \mathrm{~W}$ & $20 \%$ Duty cycle \\
Peak power L Band & $1.6 \mathrm{~kW}$ & At IF output \\
X Band NF & $3.5 \mathrm{~dB}$ & At IF output \\
L Band NF & $6.3 \mathrm{~dB}$ & \\
Max PRF & Depends on blind range and & \\
Max Pulse length & amplifier duty cycle & \\
\hline
\end{tabular}

\section{A. The current state of NeXtRAD}

In the past, several hardware limitations plagued the performance of the radar. The two primary issues were due to high jitter in the PRF trigger from the timing unit, and a reliance on human intervention to set several parameters before a data take. Setting up an experiment would take several minutes, requiring a few operators to manually change individual configuration files at each node location. Furthermore, the lack of portable power sources for the passive nodes made choosing specific bistatic geometries difficult, meaning that most recordings up to this point have been quasi-monostatic. 

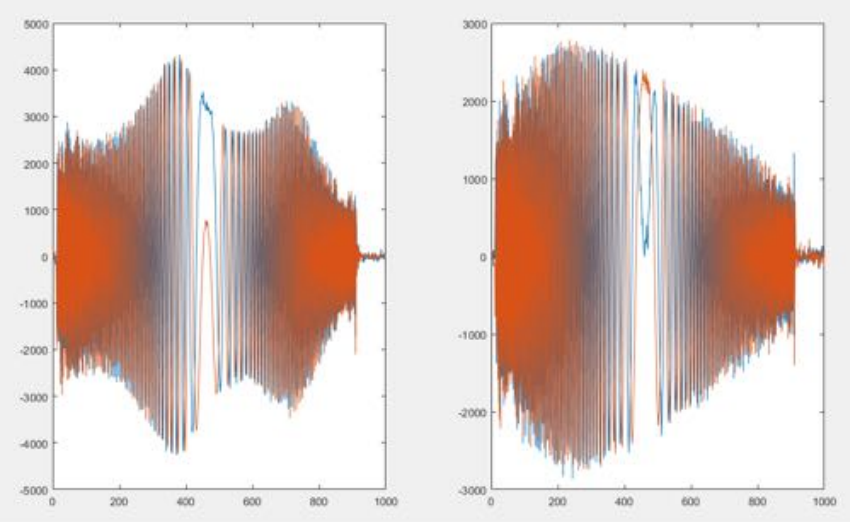

Fig. 3: RF pulse distortion through HPAs. X-band RF $10 \mu \mathrm{s}$ pulse (left) and L-Band $10 \mu s$ pulse (right).

As of the beginning of 2017, the radar has undergone several improvements, with notable examples including an FPGA and ARM based timing and control unit (TCU) implemented on a RHINO [9], [12]. The timing unit sequences the control of the power amplifiers, as well as antenna polarisation. Further more, it sets up transmit frequency, as well as the transmit waveform to be used for each PRI, enhancing system flexibility.

Experiment automation uses the local area network setup between radar nodes to transfer configuration files (see Section II). Small improvements required for the mobility of the receivers include adding $3 \mathrm{~m}$ masts for the GPS and WiFi antennas, and using portable UPS's with inverters to power the passive nodes in locations without a mains power source. In the future the receivers will be ruggedised for mobility and climate control.

In its present state, the system can be rapidly deployed, with passive nodes deployed in the canopy-covered rear of a pickup, together with their power supplies. The transmit node is still non-ruggedized and is currently limited to one site for deployment, due to size, mass and the need for a more substantial power supply.

\section{B. Preliminary Test Results}

The distortion of the RF signal from the amplifier and transmitter chain was recorded using RF power couplers. In particular, the $\mathrm{X}$-band amplifiers show significant distortion, as seen in figure 3. Similar measurements of all the commonly used pulse widths have bee made, and are used for generating the matched filter for pulse compression. As part of further improvements pre-distortion waveforms are being designed to compensate for the RF chain effects of the designed waveform and produce a more idealize transmitted signal.

\section{TEST SITE GEOMETRIES}

Presently the system is located in Simon's Bay, South Africa. In this section we show some of the possible geometries for deployment around the bay. Each node requires a

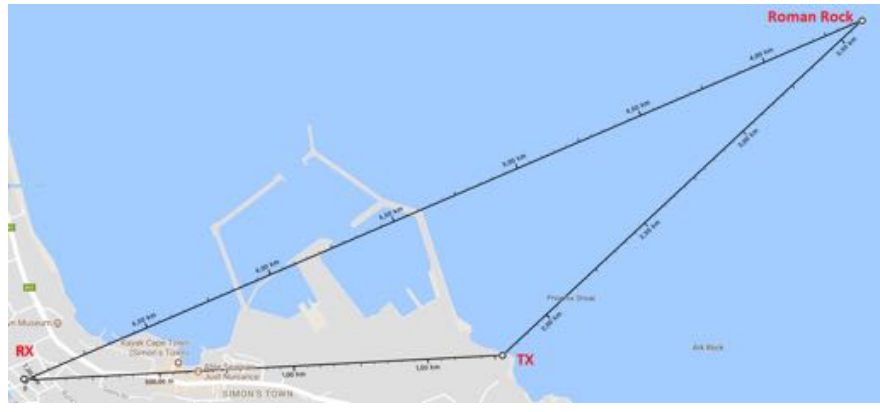

Fig. 4: Example of a bistatic geometry in Simon's Bay.

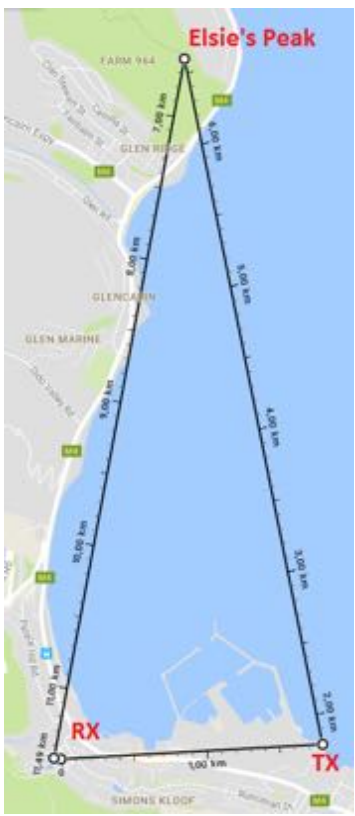

Fig. 5: Simon's Bay baselines. bistatic and forward scatter.

team of two to setup, and coordinate measurements. Figure 4 shows one typical bistatic geometry possible from our transmit site. Roman Rock is a small lighthouse used as a calibration fiduciary. Deep water in the bay away from these rocks allows for deployment of large and small vessels for tests.

Forward scatter measurements are also possible, as is shown in Figure 5. Many other multistatic configurations are possible by placing nodes along the western coastline shown in Figure 6.

For measuring monostatic and multistatic sea clutter, the geometry shown in Figure 6 is typically utilised i.e. these are the monostatic node pointing angles. The receivers (passive) are situation at two points around Simon's Bay, depending on the bistatic and multistatic angles required.

\section{TRIALS PLAN}

Micro-Doppler measurements of drone targets were carried out using a professional grade drone and licensed drone pilot. Tests are required to be within $500 \mathrm{~m}$ to $1 \mathrm{~km}$ due to local air traffic regulations. Two drone types were used, one was a smaller quadcopter and the other a large hexacopter which had 


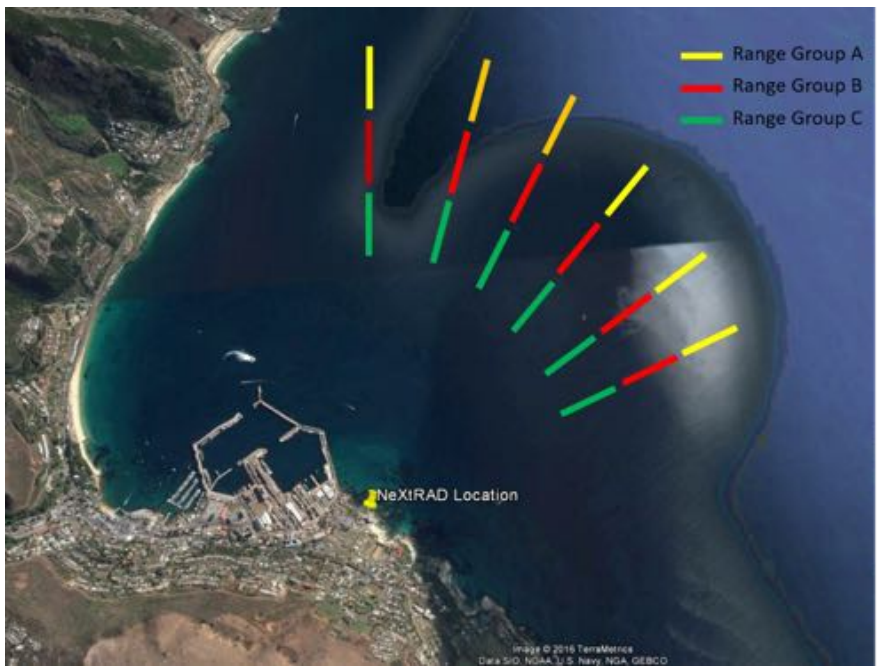

Fig. 6: Typical transmitter directions used for monostatic clutter measurements. Wind direction is from the SE, meaning that it is difficult to achieve measurements fully into the wind / waves, and also from behind the wave sets.

the ability to carry various calibration spheres. The calibration data has not yet been processed, and the drone micro-Doppler is presented in a paper submitted to the 2019 International Radar Conference in Toulon, due to space constraints in this paper.

TABLE II: Summary of December Trial Experiments

\begin{tabular}{ll}
\hline Experiment & Notes \\
\hline Multistatic Sea Clutter & $\begin{array}{l}\text { Multistatic sea-clutter recordings at various } \\
\text { bistatic angles and grazing angles }\end{array}$ \\
\hline Maritime Targets & $\begin{array}{l}\text { Recordings of maritime targets with GNSS } \\
\text { tracking for ground truth }\end{array}$ \\
\hline Forward Scatter & $\begin{array}{l}\text { Forward Scatter recordings of maritime } \\
\text { targets in Simon's bay }\end{array}$ \\
\hline Micro-Doppler with Drones & $\begin{array}{l}\text { Drone data within 500m to 1km of IMT } \\
\text { including calibration spheres }\end{array}$ \\
\hline GNSS-Denial & $\begin{array}{l}\text { NeXtRAD requires GPS for synchronisation. } \\
\text { A White Rabbit fibre-optic network is used } \\
\text { as a drop in replacement. }\end{array}$ \\
\hline
\end{tabular}

\section{RESULTS}

\section{A. Synchronisation and Timing}

NextRAD uses GPSDOs for coherent time and frequency transfer. While setting up the system, measurements between node timing and synchronisation have been made, using either the 1 pulse per second (PPS) derived from timing GPS receivers (GPSDO), or, for a single bistatic setup, from a White Rabbit fibre based 1 PPS system (WRDO) [4]-[6].

The WRDO uses a PLL with a software defined filter to generate a clean $10 \mathrm{MHz}$ reference for each node. The WRDO reduces the short term instability of the WR PPS, and improves the relative stability of the longer term averages with respect to the GPSDOs, whilst simultaneously removing the reliance

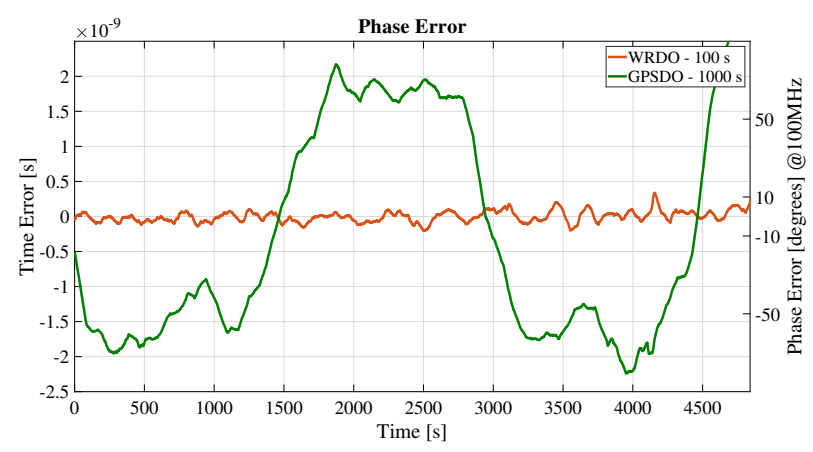

(a) Phase Difference

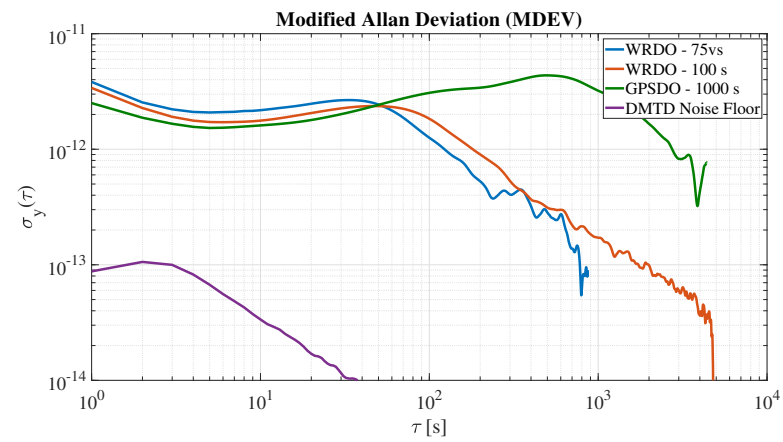

(b) Modified Allan Deviation

Fig. 7: Frequency Stability of Time and Frequency References

of the GPS carrier. The FDU within the radar multiplies this to $100 \mathrm{MHz}$ as this is the required Local Oscillator (LO) input for some sub-systems.

The relative phase stability of the $100 \mathrm{MHz}$ FDU outputs, with both GPSDO and WRDO references, was measured using a Dual Mixer Time Difference (DMTD) technique. Figure 7 shows the phase and Modified Allan Deviation (MDEV) of each reference. A PLL time constant of $100 \mathrm{~s}$ was prefered for the WR reference, having slightly improved short-term noise performance over the $75 \mathrm{~s}$ option.

The synthesiser resolution used in the passive nodes was different to the transceiver, and subsequently produced an offset LO frequency, resulting in a Doppler offset between monostatic and bistatic data of a few $\mathrm{Hz}$. This offset is removed as a phase ramp from recorded data.

\section{B. GNSS-Denial}

Concerns over GNSS denial are prevalent in modern distributed sensing systems. We investigated the effect of the loss of synchronisation of a GNSS carrier. The GNSS antenna for the monostatic node is removed after approximately 4 minutes. As seen in figure 8 , the phase difference between the references rapidly drifts, causing the bistatic node's range to drift by nearly 300 range bins $(900 \mathrm{~m})$ within 7 minutes. GNSSDO with improved holdover performance, such as those based on an atomic reference like rubidium, will reduce the rate of this effect, but will still incur an impact on the multistatic system if GNSS is compromised. Alternate synchronisation systems, 
such as the WRDO approach, above offer an effective means to avoid GNSS-Denial.

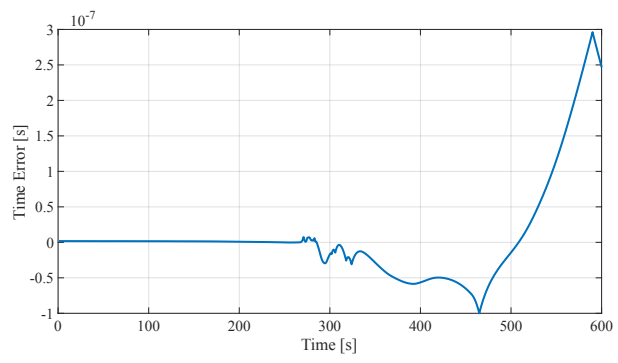

(a) DMTD Time Error
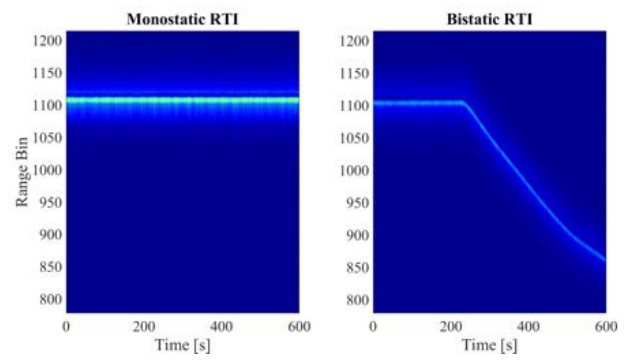

(b) Range-Time Intensity

Fig. 8: GNSS-Denial During operation, observing Roman Rock at a PRF of $99 \mathrm{~Hz}$. Bistatic range drifts approximately $900 \mathrm{~m}$ in 7 minutes.

\section{Multistatic micro-Doppler}

This subsection presents the first results obtained by analysing the maritime target data collected. For each dataset, the range cells containing the targets, either boat or drone, were separated by exploiting the additional information collected by the GPS loggers mounted on each target (Figure 9).

Figure 10 shows examples of simultaneous monostatic and bistatic micro-Doppler signatures of the RHIB at $\mathrm{X}$ band and horizontal polarization. For each range cell containing the boat radar returns, we applied the Short Time Fourier Transform (STFT) with a moving window of 200 samples, corresponding to $200 \mathrm{~ms}$, using 1024 frequency samples. In the illustrated case, the bistatic node was located at Lower North, at a distance of about $2.7 \mathrm{~km}$ from the monostatic node, and the boat was moving around a buoy located at about 540 $\mathrm{m}$ from the monostatic node and $2.64 \mathrm{~km}$ from the bistatic node, whereas the bistatic angle of the target was about 87 degrees.

From Figure 10a we observe that the signal-to-noise ratio (SNR) is roughly between $10 \mathrm{~dB}$ and $20 \mathrm{~dB}$ for the monostatic data, OdB - $10 \mathrm{~dB}$ for the bistatic data. The spectrograms show that the target Doppler frequency is initially positive for both the monostatic and bistatic data, then decreases to about -250 $\mathrm{Hz}$ in 14 seconds, and increases more rapidly to $400 \mathrm{~Hz}$ for the monostatic data and $200 \mathrm{~Hz}$ for the bistatic data, respectively. The presence of sea clutter, probably mixed with the wake of the boat, is evident only in the monostatic data, centered around $-50 \mathrm{~Hz}$, with a Doppler width of about $100 \mathrm{~Hz}$. We

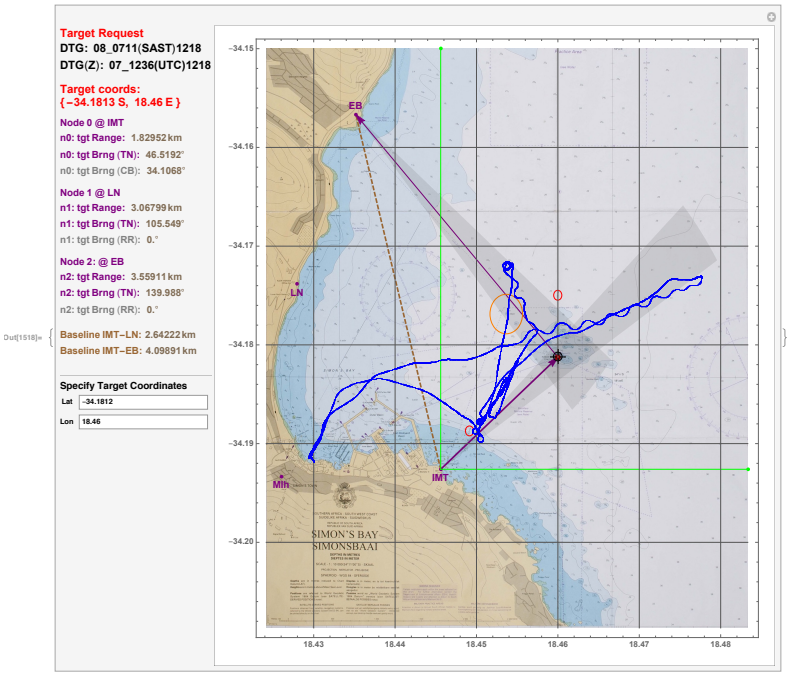

Fig. 9: GPS instrumented target in relation to bistatic beam.

note that the wake component becomes dominant over the sea clutter at about 14 seconds, i.e. the moment when the boat seems to accelerate.

Spurious wideband components are also evident in both the datasets, and proper cancellation algorithms will be used in future to attenuate them. In general, monostatic data shows higher signal and clutter power with respect to the bistatic data, meaning that different detection algorithms are needed to extract the target returns from the monostatic and bistatic data, respectively [13].

\section{CONCLUSIONS}

The Dec 2018 trials were very successful, with TBs of data captured over a two week period. Initial teething problems and equipment failures were resolved, and with experience, the operator teams were able to deploy the system quickly. Some of the data has been analysed in the month since the trials, and is presented in this paper. Although a great deal more analysis needs to be carried out.

The NeXtRAD database will eventually be available to the research community, on application to the authors. The data is hosted on a server to make access easier. The repository includes stubs of code to make basic processing easier. The data is stored in archives in the HDF5 format, accessible to most programming languages. We also seek cooperation with other research groups in the hardware of the system.

We have calibration sphere data to examine the sensitivity of the system: full polarimetric calibration, especially in the bistatic configuration is for future work. Our present site makes illumination of polarimetric reflectors difficult, and the proper calibration will be carried out once the system in more mobile.

The cost of high power TR switches being prohibitive, we will investigate fitting further power amplifiers to allow for full polarimetry on the fly. Hybrid polarimetry by transmitting circular polarisation is another possibility.

The system requires more weatherproofing and ruggedisation, to enable more measurements of land clutter and 


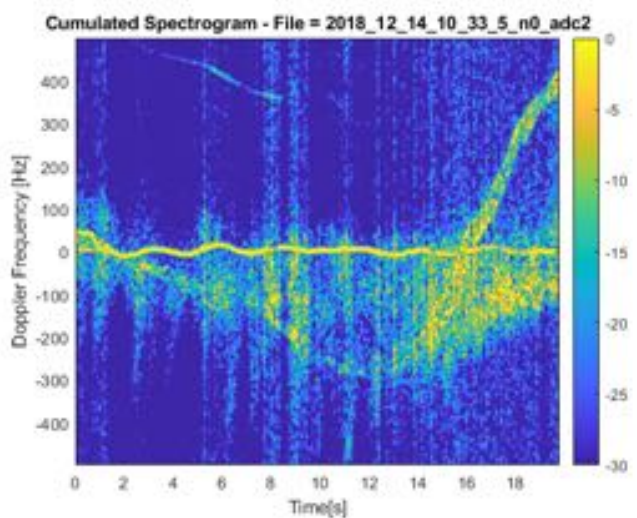

(a)

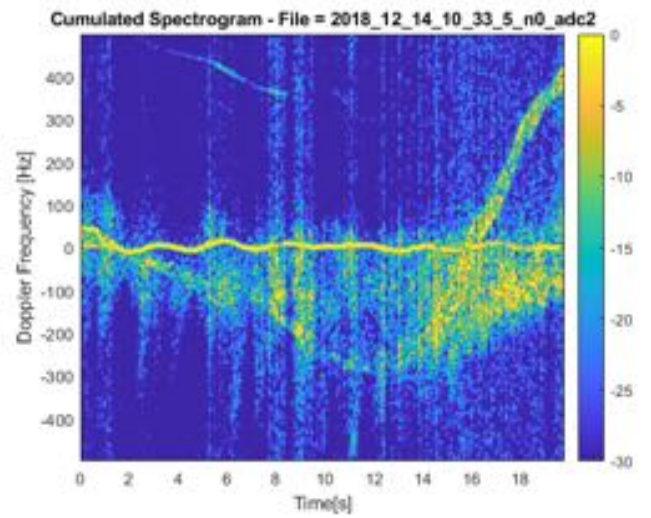

(b)

Fig. 10: Normalized spectrograms of $\mathrm{X}$ band monostatic (a) and bistatic (b) radar signatures of the RHIB, obtained from 10 range cells. Data collected on 14th December 2018, bistatic baseline of $2.7 \mathrm{~km}$.

aircraft. We also will investigate radar / communications symbiosis [14]) using the flexible waveform generator to send and receive pulses that are coded data.

\section{ACKNOWLEDGEMENTS}

The authors would like to thank Francesco Fioranelli, Adrian Stevens, Bradley Kahn, Dane Du Plessis, Jacques Cilliers, Shirley Coetzee, Stephan Sandenbergh, Terje Johnsen, William Miceli, Randy Cheng, Jacques Cilliers for the support provided during the measurement campaign, and the Office of Naval Research Global (USA), the Institution of Engineering and Technology, FFI and SA National Defence Force for the funding provided.

\section{REFERENCES}

[1] Tom Derham, K Woodbridge, H Griffiths, et al. "The design and development of an experimental netted radar system". In: Radar Conference, 2003. Proceedings of the International. IEEE, 2003, pp. 293-298.
[2] Michael Inggs, Hugh Griffiths, Francesco Fioranelli, et al. "Multistatic radar: System requirements and experimental validation". In: Radar Conference (Radar), 2014 International. IEEE, 2014, pp. 1-6.

[3] J. S. Sandenbergh and M. R. Inggs. "A common view GPSDO to synchronise netted radar”. In: IET 2007 International Conference on Radar Systems. Oct. 2007, pp. 1-5. DOI: 10.1049/cp:20070499.

[4] JS Sandenbergh, MR Inggs, and WA Al-Ashwal. "Evaluation of coherent netted radar carrier stability while synchronised with GPS-disciplined oscillators". In: Radar Conference (RADAR), 2011 IEEE. IEEE, 2011, pp. 1100-1105.

[5] JS Sandenbergh and MR Inggs. "Synchronizing network radar using all-in-view GPS-disciplined oscillators". In: Radar Conference (RadarConf), 2017 IEEE. IEEE, 2017, pp. 1640-1645.

[6] S Lewis, JS Sandenbergh, and MR Inggs. "Evaluating an off-the-shelf white rabbit system to synchronise network radar via optic fibre". In: Radar Conference (RadarConf), 2017 IEEE. IEEE, 2017, pp. 1657-1662.

[7] M Inggs, Alessio Balleri, WA Al-Ashwal, et al. "NetRAD multistatic sea clutter database". In: Geoscience and Remote Sensing Symposium (IGARSS), 2012 IEEE International. IEEE. 2012, pp. 2937-2940.

[8] D. Jordan, M. Inggs, and D. O’Hagan. "NeXtLook a lightweight, real-time quick-look processor for $\mathrm{NeX}$ tRAD”. In: International Conference on Radar Systems (Radar 2017). Oct. 2017, pp. 1-6. DOI: 10.1049/cp. 2017.0470.

[9] M. Inggs, D. Jordan, and T. Learner. "Through the clouds: Exploring the potential of infrastructure-as-aservice for radar". In: International Conference on Radar Systems (Radar 2017). IET, Oct. 2017, pp. 1-6. DOI: $10.1049 / \mathrm{cp} .2017 .0447$.

[10] Ansible Website. https://www.ansible.com/.

[11] Coffee Can Radar. https ://spectrum. ieee.org/geeklife/hands-on/coffeecan-radar.

[12] M. Inggs and A. van der Byl. "NeXtRAD and RHINO radar: Harnessing the herd for networked radar". In: 2014 International Radar Conference. 2014, pp. 1-5. DOI: 10.1109/RADAR.2014.7060408.

[13] Riccardo Palamà, Maria Greco, and Fulvio Gini. "Multistatic adaptive CFAR detection in non-Gaussian clutter". In: EURASIP Journal on Advances in Signal Processing 1 (Oct. 2016), p. 107. ISSN: 1687-6180. DOI: 10.1186/s13634-016-0408-1. URL: https://doi.org/10. 1186/s13634-016-0408-1.

[14] Michael Inggs and Amit Mishra. "A New Taxonomy for Symbiotic EM Sensors”. In: CoRR abs/1702.07928 (2017). arXiv: 1702.07928. URL: http://arxiv.org/abs/ 1702.07928 . 